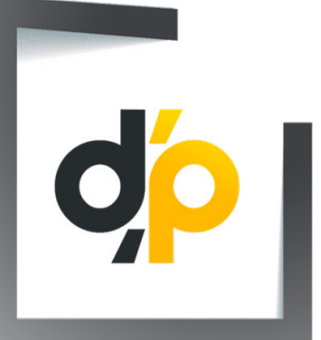

d.perspectivos siglo XXI

VOLUMEN 7 NÚMERO 13

3 DE MARZO DEL 2020
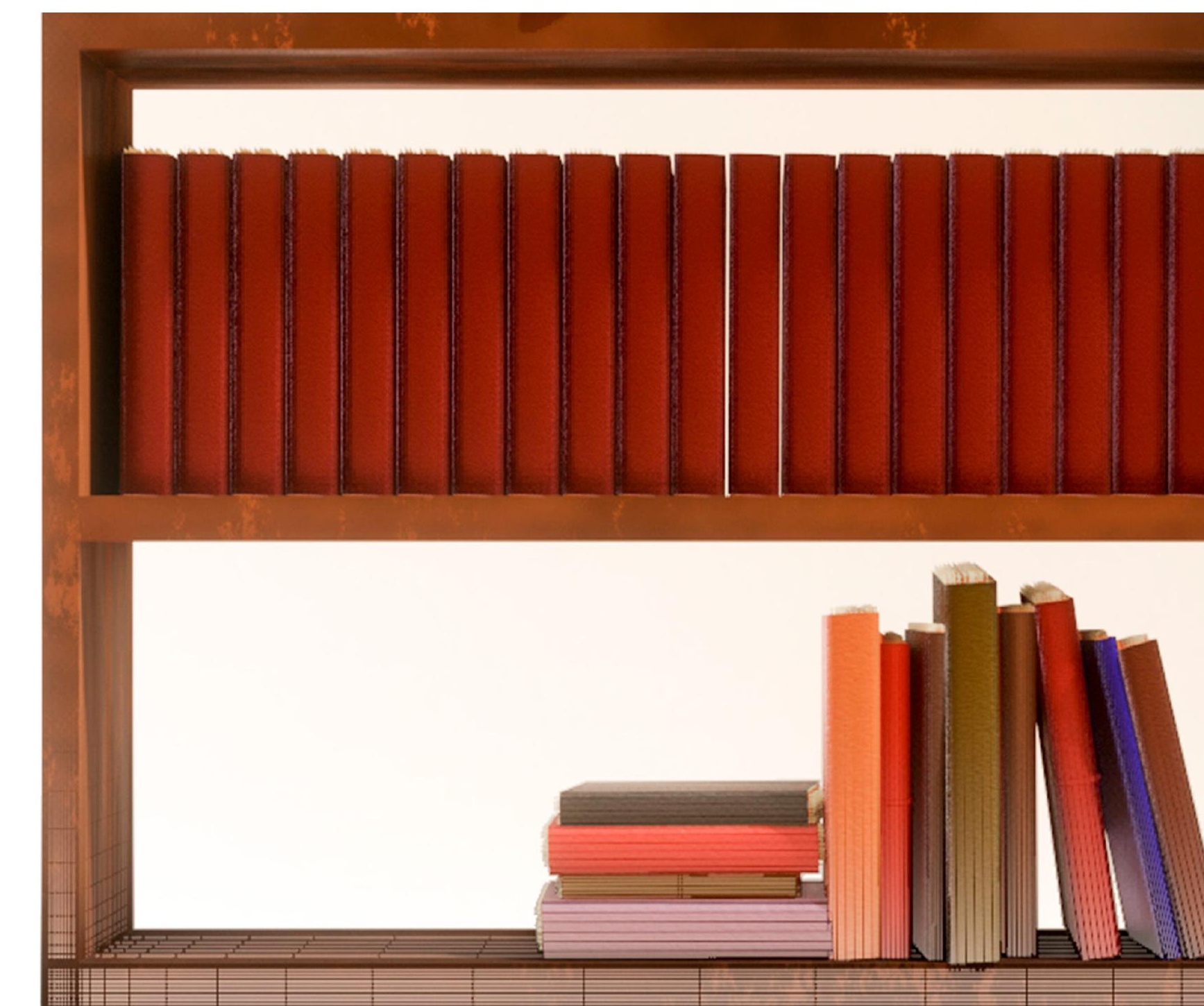

章

4

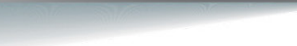




\section{El uso de las TIC en la enseñanza de las matemáticas y su importancia en el desarrollo de capacidades digitales para el campo laboral}

\section{The use of ICT, in the teaching of mathematics and its importance in the development of digital skills, for the labour market}

Emilio David Olvera Rebolledo

Maestro en Estudios Sustentables Regionales y Metropolitanos

Universidad Autónoma del Estado de México
Reyna Cardoso Malaquias Maestra en Humanidades: Ética Campus Universitario Siglo XXI Contacto: biblioiipac@hotmail.com

Recepción: 18/10/2019

Aceptación: 06/12/2019

DOI: http://doi.org/10.53436/21ak3O8V

\section{Resumen}

Actualmente, el uso de las Tecnologías de la Información y Comunicación (TIC) es un elemento obligatorio para la enseñanza en todos los niveles de educación, particularmente para el nivel superior. Esto implica que en la formación profesional se tenga que atender la demanda de capacidades específicas para el mercado laboral, ya que apuntan cada vez más al trabajo independiente con el apoyo de esos recursos En este documento se exponen los resultados obtenidos de una muestra no probabilística, aplicada a tres grupos de quince alumnos, al implementar estrategias de aprendizaje autónomo en la conducción de asignaturas con carga en matemáticas, para licenciaturas económico-administrativas impartidas en el Grupo Educativo Siglo XXI, en las que se emplearon herramientas electrónicas, y plataformas digitales para el desarrollo del programa académico. De esta experiencia, se señala que el apoyo didáctico que representan las TIC, favorece las habilidades para la solución de problemas matemáticos, correspondientes a la investigación y al manejo de información, al pensamiento crítico y a la toma de decisiones. Se identifica la figura del docente como un acompañante en la construcción del conocimiento. Bajo una perspectiva cualitativa se reflexiona sobre las capacidades que los estudiantes egresados de educación superior deben desarrollar con las TIC, lo mismo que en la relación con su impacto en el mercado laboral.

Palabras clave: TIC, Educación superior, Competencias digitales, Matemáticas, Mercado laboral. 


\section{Abstract}

Currently, the use of Information and Communication Technologies (ICT) is a mandatory element for teaching at all levels of education, particularly at the higher level. This implies that professional training has to meet the demand for specific skills for the labour market, since they increasingly aim for independent work, with the support of these resources. This document presents the results obtained from a non-probabilistic sample, applied to three groups of fifteen students, when implementing autonomous learning strategies in the conduct of subjects with a load in mathematics, for economicadministrative degrees taught in Grupo Educativo Siglo XXI, in which electronic tools and digital platforms were used for the development of the academic program. From this experience, it is pointed out that the didactic support represented by ICT favors skills for solving mathematical problems, corresponding to research and information management, critical thinking and decision-making. The figure of the teacher is identified as a companion in the construction of knowledge. From a qualitative perspective, a relection is made on the capacities that higher education graduates must develop with ICT, as well as in relation to their impact on the labour market.

Key words: ICT, Higher education, Digital skills, Mathematics, Labour market.

\section{Introducción}

Las Tecnologías para la Información y la Comunicación (TIC) en la formación académica deben constituir un conjunto de herramientas y habilidades necesarias para comprender la migración de escenarios didácticos presenciales a remotos. A continuación, se describe la práctica docente que tuvo lugar en el Grupo Educativo Siglo XXI de la ciudad de Toluca, en el semestre 2019 B, con 3 grupos de alumnos de licenciaturas económicoadministrativas. Se exponen los resultados derivados de la enseñanza en un entorno virtual para asignaturas como estadística matemática, contabilidad y estados financieros y estadística matemática; también se menciona un conjunto de recomendaciones relacionadas con la aplicación de plataformas electrónicas de aprendizaje, paquetería, dispositivos, aplicaciones gratuitas y recursos empleados para interactuar con los estudiantes que se encuentran a distancia, todo con el objetivo de favorecer su aprovechamiento académico.

El hardware y software que se empleó por su mayor disponibilidad para los alumnos se seleccionó por las características de portabilidad y fácil 
reproducción, ya que permite que sean herramientas de apoyo importante para la recuperación y consulta de la información, además de que favorecen el trabajo independiente del alumno. Los medios de comunicación que se usaron fueron Quicktime player, Microsoft Teams y Skype, y las aplicaciones en iPad fueron principalmente Microsoft Onenote y Notability.

En la enseñanza de asignaturas de contenidos relacionados a matemáticas, el uso del pizarrón en el aula de clases es casi obligatorio, ya que el docente puede explicar en él fórmulas y ecuaciones o resolver problemas frente al grupo, de este modo, el estudiante consigue observar la manera de plantear y operar un problema, desarrollarlo y asimilar sus resultados. Sin embargo, se requiere implementar nuevas herramientas educativas y, se demanda aún más, aplicar estrategias dinámicas. Por eso, para este estudio se empezaron a usar diapositivas o notas elaboradas en equipos de cómputo con procesadores de texto como Microsoft Word, también se recurrió a paquetería especializada como LaTex, lo cual requiere de un tiempo de preparación mucho mayor a lo habitual, que en ocasiones sobrepasa la duración de la misma clase, no obstante, es una alternativa adecuada para compartir el contenido con los alumnos, publicarlo o entregarlo digitalizado.

Se observó que LaTex favoreció el aprendizaje, ya que posee diversos servicios y aplicaciones que convierten a este sistema en una alternativa útil para presentar textos matemáticos con un aspecto profesional. El índice, la numeración de las páginas, los encabezados de tablas, los cuadros, las figuras, así como el control minucioso de las referencias bibliográficas se generan automáticamente, aunque la curva de aprendizaje de dicho lenguaje de composición tipográfica es más lenta en comparación al procesador de textos.

La herramienta tradicional del pizarrón no es muy viable para impartir las distintas clases de manera virtual, pero el tiempo requerido para exponer todas las notas a través de un procesador de textos es demasiado, por lo que después de algunos intentos, se descartó dicha opción. Para dar solución a lo anterior se empleó la aplicación Quicktime, con ella se grabaron videos de las notas explicando el desarrollo de tareas y la resolución de problemas de manera manuscrita utilizando Apple Pencil. A través de la aplicación de Microsoft Teams, se empleó como principal recurso la pizarra electrónica, en la que, con Apple Pencil, se explicó el contenido de la clase. A su vez, la sesión fue grabada por los alumnos para que se encontrara disponible para su 
posterior consulta en esta plataforma.

Las sesiones de dudas se programaron a través de Skype, se organizaron en pequeños grupos y durante su desarrollo se empleó el iPad, junto con Apple Pencil para realizar los ejercicios de manera manuscrita. Además, se compartió la pantalla a través de la aplicación Quicktime player, de esta manera, los alumnos podían visualizar el proceso y la resolución de problemas en tiempo real a través de la aplicación Notability, para después convertir el archivo a formato PDF.

Schoology es la plataforma educativa que se empleó como escenario virtual para la impartición de los cursos, ya que su ágil acceso y facilidad de instalación permitió que los alumnos entendieran en las primeras sesiones la dinámica de trabajo. Por sus características de red social, se consideró una herramienta eficiente para mantener actualizados los avisos, llegar a acuerdos y discutir asuntos conjuntos a través de foros, de tal forma que cubrió las necesidades de comunicación entre el docente y el grupo.

La mayoría de los alumnos reaccionaron de manera favorable ante las nuevas implementaciones para el desarrollo del programa académico. La interacción de las primeras tareas grupales dio oportunidad de identificar a los alumnos que ya poseían conocimiento sobre el uso de las herramientas propuestas. El docente ocupó mayor tiempo para el diseño de actividades encaminadas a disminuir la brecha de conocimiento en el uso de las TIC entre los integrantes de cada grupo y para incrementar la atención personalizada, así mismo, se identificó la necesidad de capacitación constante.

\section{Desarrollo}

Las herramientas tecnológicas que se seleccionaron por su eficiencia y apoyo para la comunicación entre profesor y alumno son Quicktime player, Microsoft Teams y Skype, pero además de éstas, es fundamental que los estudiantes cuenten con un espacio que facilite su acceso a la información de la clase para que puedan recurrir a ella y aclaren dudas, es decir, es necesario un lugar apropiado para depositar información de consulta, ya sea de manera previa o posterior a la clase. También se necesitan los registros y el control de las actividades del grupo, para ello el principal recurso que se empleó en el trabajo con los estudiantes a distancia fue Schoology. A continuación, se describe cómo esta plataforma permitió desarrollar los ejercicios y exponer los contenidos de las asignaturas. 
Se podría considerar que Schoology es un sistema de gestión de aprendizaje del tipo Management System Learning (LMS, por sus siglas en inglés). Ruíz (2015) desde su blog Planificación curricular afirma: "es una propuesta para gestionar mejor el aprendizaje a través de la integración de herramientas digitales en la nube. Una plataforma que promete convertirse en la red social de la educación". En el sitio oficial de Schoology (2020) se especifican las actividades que se pueden desarrollar, en la experiencia de uso las más empleadas fueron las siguientes: gestión de los cursos impartidos, publicación de anuncios y actualizaciones de contenido, carga de archivos (incluyendo videos de clases grabadas), diseño de exámenes en los que es posible crear bancos de preguntas con texto enriquecido y ecuaciones, control de asistencias y control de calificaciones.

Los principales elementos que se tomaron en cuenta para seleccionar esta plataforma fueron, su versatilidad, su disponibilidad -ya que es totalmente gratuita- y su alto grado de compatibilidad con distintos dispositivos electrónicos, pues únicamente se requiere de un navegador web para acceder al contenido. Otro de los elementos valorados para su implementación fue el control y seguimiento cercano de los alumnos; con Schoology, en un principio, se necesita crear grupos virtuales para que los estudiantes se unan a ellos a través de un código, que podrá ser compartido a través de correo electrónico, y una vez que ingresen tienen para su consulta todo el curso e, incluso, pueden descargar las actividades en sus celulares, tablets o laptops para que les sea posible revisar los elementos vistos con anterioridad, cuando se termine el ciclo escolar.

Las actividades con el uso de las TIC requieren de una plataforma intuitiva para el profesor y el alumno, en la que sea visible en una misma página y de modo sencillo el contenido del curso, las secciones de avance, las actualizaciones, el control de asistencia individual, las calificaciones y también las tareas o exámenes próximos, para identificar de una forma general los recursos y las actividades programadas. Se sostiene que la herramienta Schoology es un apoyo para optimizar las labores docentes, ya que una de las actividades que se considera más demandante en cuanto a tiempo es la revisión de tareas, $\mathrm{y}$ en este aspecto permite descargar por lote todas y cada una de las entregas que los alumnos hayan realizado; así, se pueden efectuar las respectivas anotaciones, comentarios, correcciones y asignación de calificaciones. 
Por otra parte, la gestión calendarizada del contenido contribuye a visualizar si los estudiantes realizan las entregas de tareas en tiempo, o bien con retraso. En particular, la manera en la que la plataforma permite a los estudiantes realizar la entrega de tareas se considera útil.

Cabe mencionar que para el diseño de los exámenes se tiene la alternativa de emplear los siguientes formatos de preguntas: falso o verdadero, opción múltiple y ordenamiento, en los que tanto el profesor como el alumno conocerán el resultado de la prueba de manera inmediata de acuerdo con los criterios de evaluación que se definieron para el curso. En este sentido, es una gran ventaja programar la visibilidad de archivos, tareas y exámenes, ya que desde esta herramienta se configura una fecha de inicio o de término para cada uno de los contenidos del curso, esto se vuelve bastante útil cuando se tiene una planeación ajustada y se pretende que el contenido temático de la unidad de aprendizaje sea visto por los estudiantes en momentos específicos.

En cuanto a la programación de los exámenes, es posible asignar hora y fecha, duración, número de intentos que tendrá el estudiante para contestarlo, con la opción de que cada una de las pruebas sea única y diferente, ya que los reactivos que las componen se extraen de un banco de preguntas, además permite contestar en el orden deseado, de modo que, si se pospone resolver una cuestión, se puede regresar a ella más tarde. Es importante agregar que también se habilita una casilla de comentarios para que el estudiante incorpore mayor información u otros elementos a su respuesta.

Para la asignación de calificaciones, la plataforma permite al profesor visualizar todos los puntajes de los trabajos y las tareas de los alumnos, así como el puntaje obtenido en los exámenes, de esta manera es sencillo disponer de la información relacionada con el aprovechamiento de los estudiantes. En particular, una de las opciones más útiles del control de calificaciones es la posibilidad de editarlas manualmente para los casos en que sea necesario incorporar mayor flexibilidad en la manera de trabajar, por ejemplo, en algunas ocasiones se requiere considerar las posibles dificultades de conectividad que pudieran tener los estudiantes.

Sin duda, Schoology es un recurso que ayuda en la gestión del curso, ya que se adapta a las necesidades de los alumnos; en cuanto al acceso a la información, es un apoyo importante, pues registra calificaciones, tareas, asistencia y control de trabajos entregados en tiempo y forma, además el alumno visualiza sus progresos en un espacio abierto permanente para externar sus dudas. 
Las características de esta plataforma y las necesidades que cubre en el desarrollo de las sesiones de cada asignatura son un ejemplo de cómo las TIC ayudan en la apropiación de las competencias digitales. Éstas, de acuerdo con Ferrari (2012, citado en Terreni et al., 2019, p. 67) son:

El conjunto específico de herramientas y aplicaciones que se requieren cuando se utilizan las TIC y los medios digitales para realizar tareas, resolver problemas, comunicar información, gestionar la información, colaborar, crear y compartir contenidos; y construir un conocimiento eficiente y eficaz, de manera crítica, autónoma, reflexiva, para el trabajo, el ocio, la participación, el aprendizaje y la socialización.

A partir de lo anterior, es pertinente reflexionar sobre la transformación de los roles de docentes y dicentes: en ambos casos se requiere desarrollar habilidades como la resolución de problemas, la toma de decisiones, la creatividad y la innovación para el manejo de las TIC. Ante una mayor apertura a los medios de información, con recursos que están cada vez más al alcance de los usuarios (por ejemplo, a través de la recuperación de datos desde plataformas digitales académicas, con software certificado de acceso abierto, o consultas en redes de especialistas) estas prácticas son una oportunidad para que alumnos y maestros incrementen su autoaprendizaje.

Quintero y Jerez (2019) sugieren que el nivel medio superior es el momento "donde el trabajo con las TIC se torna más importante, porque desarrollan ciertos puntos claves para hablar del estudiante como protagonista de su aprendizaje, ya que aumentan la motivación a la hora de despertar interés por aprender y comprender" (p. 21). En el caso de los docentes, su formación profesional para el dominio de las TIC, también deberá ser una tarea de instrucción independiente; tal como asevera el texto de González et al., (2017) en el análisis sobre las competencias en el uso de las TIC por parte de los profesores universitarios en nuestro país: “...en el contexto de las universidades en México, es relevante abordar las limitantes de los programas formativos que buscan desarrollar las competencias tecnológicas en el profesorado..." (pp. 6-7). Si no están dadas las condiciones de capacitación, $\mathrm{y}$ ante las nuevas dinámicas en el proceso de enseñanza aprendizaje, profesores y alumnos tendrán que empeñarse aún más en la apropiación de conocimientos. 
En la interacción entre los recursos para el aprendizaje que ofrecen las TIC y su accesibilidad, se puede identificar la función del docente frente a sus alumnos como asesor para la gestión de la información, en la obtención de contenidos pertinentes para la formación académica está también fuera del aula y es responsabilidad igualmente del alumno. El ejemplo que da Sucerquia et al. (2016) sobre la paquetería de "software interactivos para el intercambio de información y la constitución de comunidades de aprendizaje en el campo de la educación matemática que se están empleando en diferentes redes y se proyectan como un espacio para la creación de conocimiento colectivo" (p.43) ilustra la apertura para integrar nuevas manifestaciones en los procesos de enseñanza. Quintero y Jerez (2019) afirman desde su experiencia en el nivel medio superior que:

Los educandos tienen la oportunidad hoy día de ampliar su experiencia desde el aprendizaje constructivista, sin tener la excusa de no poseerla, porque en la gran mayoría de los países los estudiantes de bachillerato cuentan con una computadora. Además de esto, estas herramientas le ofrecen opciones para lograr convertir el aula tradicional en un nuevo espacio, poniendo a su disposición actividades innovadoras de carácter colaborativo y con aspectos creativos que les permitan afianzar lo que aprenden de forma amena y divertida. (p. 22)

Para Rué, J. (2016) es necesaria "Una formación orientada hacia el procesamiento de la información que requiere de una atención mucho mayor por parte de los docentes hacia los estudiantes que aquélla fundada en la simple transmisión y acumulación de la información" (p. 100). En el ejercicio de conducir a los alumnos de Grupo Educativo Siglo 21 hacia las herramientas comunicativas que ofrecen las TIC (guiando sus progresos particulares), el uso de Microsoft Teams y Skype permitió sostener comunicación de manera directa, identificando necesidades de orientación académicas para los alumnos.

Ahora se busca crear una estrategia cuidada en la que es fundamental "seguir cultivando el gusto por aprender a aprender a través de la incorporación de nuevas herramientas tecnológicas acordes a las nuevas generaciones de alumnos y al contexto actual de información y transformación social" (Abascal y López, 2017, p. 64). Con la intención de que el docente fomente la investigación en las asignaturas relacionadas a matemáticas tendrá que mantener motivados a sus alumnos en la búsqueda continua de conocimiento nuevo para obtener mayor dominio en los temas de su plan académico, para su formación profesional y su futuro como egresado. 


\section{Las TIC en la educación superior y su importancia para el campo laboral}

La inserción laboral de los futuros egresados universitarios está directamente relacionada con su dominio sobre las TIC, dado que éstas representan una habilidad diferenciadora y competitiva en el entorno globalizado para el que se debe preparar desde la formación académica. Nos enfrentamos a nuevas maneras, de aprender y enseñar:

En el siglo XXI, el empleo de las TIC ha representado un cambio significativo en la educación, debido a que ha propiciado una visión dirigida a que el empleo de éstas genere una transformación de la Sociedad de la Información (SI) para que se promueva la Sociedad del Conocimiento (SC), lo cual implica un desafío en la integración de las tecnologías desde un enfoque educativo, pedagógico e innovador. (Licona y Veytia, 2019 p. 94)

Con el ejercicio de implementación de las TIC para la enseñanza de las matemáticas, correspondientes a las asignaturas económico-administrativas del ciclo 2019 B en el Campus Universitario Siglo XXI, se motivó a los alumnos a trabajar de manera independiente y a su propio ritmo, se observó el trabajo colaborativo entre compañeros: algunos manifestaron sentirse acompañados en el cumplimiento de sus tareas, con el uso de los recursos digitales sugeridos; además, indicaron que a través del acceso a diferentes formatos, cada uno va desarrollando su propio estilo de aprendizaje.

Es importante considerar que el estudiante de educación superior se está preparando para escenarios en los que se requiere su intervención en la resolución de problemas en entornos en los que deberá ser capaz de afrontar las exigencias de su ámbito laboral, el uso de las TIC para la resolución y comprensión de las matemáticas en asignaturas económico-administrativas en nivel licenciatura implica un acercamiento a su futura actividad profesional.

La investigación de Torres y Ochoa (2018) asevera que la disparidad económica en nuestro país está directamente relacionada con el empleo de las tecnologías y que "representa un elemento de diferenciación salarial tan importante como lo es la escolaridad" (p. 361). Esto permite identificar que los docentes se encuentran frente a un reto mayor, ya que deben coadyuvar a la formación de personas capacitadas para tener un buen desempeño académico 
y profesional, por ello la construcción de las competencias digitales deberá ser una práctica constante, conforme a las necesidades del mercado laboral.

Las modificaciones necesarias que ahora se realicen en las prácticas de la educación superior tendrán un impacto determinante en el futuro laboral de los egresados, así lo manifiesta José Navarro Cendejas (2017) en la introducción de su texto Educación superior y trabajo: hacia la construcción de un sistema de información sobre egresados. En él señala que las instituciones de educación superior "necesitan tener el pulso del mercado laboral para verificar la vigencia de sus planes y programas de estudio, y determinar si las habilidades y conocimientos desarrollados por sus estudiantes están siendo valorados por el sector productivo" (p. 4). La relación entre empresas y universidad debe ser cuidada por la institución de educación superior, en vías de que sus egresados tengan una inserción laboral satisfactoria.

Becerril (2020), por su parte, destaca: "las competencias y/o habilidades digitales propuestas por el gobierno mexicano para promover la adopción de las TIC se enfocan en la tecnología y no toman en cuenta ni las condiciones del mercado laboral ni los intereses de los actores involucrados" (p. 89).

Ante la falta de políticas públicas estructuradas en relación a las TIC para la integraciónal empleo profesional de los egresados de licenciatura, la formación académica universitaria deberá atender los requerimientos por parte de los empleadores y las competencias digitales adquiridas en el desarrollo de sus programas. No obstante, identificar la inserción laboral es una dificultad ya que "los seguimientos de egresados se realizan con metodologías diversas, con resultados desiguales y suelen ser poco transparentes de cara a la sociedad" (Navarro, 2017, p. 6). Dado lo anterior, se tendrá que comprometer cada universidad a identificar que sus egresados estén ocupando puestos profesionales relacionados a su formación académica, de manera que prevean convenios de inserción laboral con instituciones y empresas.

El economista Roberto Newell (2018) señala sobre la relación entre la actividad económica y quienes están empleados en ella que: "La eficiencia con que opera una economía está estrechamente ligada al funcionamiento de su mercado laboral. Cuando un mercado laboral funciona adecuadamente, los trabajadores se emplean en ocupaciones que les permiten aprovechar al máximo sus destrezas y habilidades" (párr. 3). En contraparte, un mercado laboral ineficiente implica niveles bajos de productividad, pobreza multidimensional, informalidad laboral, aunado a la limitada movilidad de 
los trabajadores hacia posiciones con mejores salarios (Varela y Oceguera, 2020, p. 113), dicha situación se considera uno de los principales elementos a resolver debido a los nuevos retos económicos y laborales que la población debe enfrentar como resultado de la globalización.

En el campo laboral se manifiesta una clara repercusión que divide a quienes tienen acceso a las tecnologías de quienes no han logrado desarrollar capacidades digitales como factor competitivo. Tendrán mayor posibilidad de ocupar un empleo más remunerado quienes adquieran una mejor capacidad de adaptarse a las necesidades que el mercado del empleo requiere. En consecuencia, es necesario que los individuos tengan la oportunidad de mejorar esas habilidades y conocimientos, ya que el capital intelectual del que dispongan les permitirá incorporarse en actividades productivas de manera más flexible. Se debe ponderar la importancia del uso de las TIC en la formación académica, en la vida cotidiana y, por supuesto, en el desempeño profesional, en palabras de Abarca (2015): "promover el desarrollo integral de los estudiantes universitarios para poder ser competentes y efectivos en una sociedad en la cual el conocimiento y la tecnología van de la mano en una misma ruta" (p. 348). Abarca hace un análisis sobre la motivación y frecuencia en el uso de las TIC en el que identifica una actitud positiva de parte de los docentes para trasladar sus prácticas de enseñanza tradicional a dinámicas de mayor innovación. Encontrarse ante un grupo de alumnos como facilitador de recursos para el aprendizaje, permite aprender continuamente no sólo sobre una materia, sino sobre la interacción humana para darle un mayor valor a la vocación docente.

\section{Conclusiones}

Ante las nuevas necesidades, tanto en la docencia como en el contexto profesional, nos enfrentamos a panoramas de aprendizaje más significativos que deberán ser útiles para su aplicación en la vida laboral y cotidiana. La tecnología tiene presencia en todos los escenarios, el ejemplo de la enseñanza de asignaturas relacionadas a las matemáticas con el empleo de las TIC es muestra de ello. En el caso referido: los temas desarrollados, las evaluaciones y las evidencias de actividades asignadas pudieron gestionarse de manera óptima tanto para los alumnos como para el docente. La retroalimentación se dio de manera individualizada, ya que se proporcionaron medios de comunicación más directos como el WhtasApp, FaceBook y mensajes por medio de la plataforma Schoology, lo cual amplió el escenario del salón de clase a los dispositivos de los dicentes. Las Tecnologías de la Información y la 
Comunicación han modificado las formas de conducirnos en distintas esferas y la educación no ha quedado al margen; cambiar del pizarrón colgado en la pared a al uso de pizarra electrónica es el ejemplo de los ajustes necesarios para transformar la enseñanza. En la experiencia académica aquí expuesta, el apoyo de una plataforma como Schoology permitió poner a disposición los documentos de consulta y las sesiones grabadas para dar soporte a los alumnos en caso de que no pudieran ingresar al momento de la clase. Las habilidades digitales adquiridas en la formación universitaria son un punto focal de reflexión dentro de la planeación de programas académicos de nivel superior, dado el impacto que directamente se relaciona con las necesidades del mercado laboral, el que se vuelve cada vez más competido y especifico y en el que ya no podemos pensar de manera local, sino global por la híper conectividad a Internet.

\section{Referencias}

Abarca Amador, Y. (2015). El uso de las TIC en la educación universitaria: Motivación que incide en su uso y frecuencia. Revista de Lenguas Modernas, 22(2015), 335-349. https://revistas.ucr.ac.cr/index.php/rlm/ article/view/19692.

Abascal Mena, R., López Ornelas, E. (2016). El uso de m-learning para motivar al alumno en su aprendizaje: Caso de estudio en la UAM Cuajimalpa. En Eestrategias didácticas en educación superior basadas en el aprendizaje: Innovación educativa y TIC / Carlos Roberto Jiménez González et al; Ciudad de México, México: Universidad Autónoma Metropolitana Unidad Cuajimalpa. http://ilitia.cua.uam.mx:8080/jspui/ handle/123456789/858.

Becerril Velasco C. (2020). Pobreza urbana y apropiación de las Tecnologías de la Información y Comunicación (TIC) en México. Observatorio (OBS) Journal, 14(2), 72-92. http://obs.obercom.pt/index.php/obs/ article/view/1597/pdf.

González Bello, E., López Espinosa, J., y Estévez Nenninger, E. (2017). Competencias TIC del profesorado universitario: Consideraciones para una enseñanza innovadora desde la formación docente. Revista Brasileira de Ensino Superior, 3(3), 3-22. doi: https://doi.org/10.18256/24473944.2017. v3i3.2128. 
El uso de las TIC en la enseñanza de las matemáticas y su importancia en el desarrollo de capacidades digitales para el campo laboral

Licona Meneses, K., Veytia Bucheli, M. G (2019) El empleo de las TIC en la educación superior. Educando para educar. 37. https://beceneslp.edu. mx/ojs2/index.php/epe/article/view/47.

Monroy, A., Hernández, I. A., y Jiménez, M. (2018). Aulas Digitales en la Educación Superior: Caso México. Formación universitaria, 11(5), 93-103. doi: https://dx.doi.org/10.4067/S0718-50062018000500093. Navarro Cendejas, J. (2017). Educación superior y trabajo: Hacia la construcción de un sistema de información sobre egresados. Diálogos sobre educación. Temas actuales en investigación educativa, 8 (14), 1-10. http://dialogossobreeducacion.cucsh.udg.mx/index.php/DSE/ article/view/218.

Newell, R. (18 de enero de 2013). Funcionamiento del mercado laboral. Reforma. https://www.reforma.com/aplicacioneslibre/preacceso/articulo/ default.aspx?_rval=1\&urlredirect.

Quintero, M., Jerez, J. (2019). Las Tic para la enseñanza de la matemática en educaciónmediageneral.UniversidadpolitécnicaterritorialdeMaracaibo. Revista electrónica de ciencia y tecnología del Instituto Universitario de Tecnología de Maracaibo. 6(1), 20-36. http://201.249.78.46/index. $\mathrm{php} / \mathrm{recitiutm} / \mathrm{article} / \mathrm{view} / 168 / \mathrm{pdf}$.

Rué, J. (2016). El aprendizaje autónomo en educación superior. Madrid, España: Narea Ediciones. https://elibro.net/es/ereader/cus21/45946.

Ruíz R. (15 de julio de 2015) Schoology [Schoology es una propuesta para gestionar mejor el aprendizaje a través de la integración de herramientas digitales en la nube. Una plataforma que promete convertirse en la red social de la educación] Planificación curricular. http:// ruiz3c1plancurricular.blogspot.com/?view=classic.

Sucerquia, E.A., Londoño Cano, R.A., Jaramillo López, C.M. y De Carvalho Borba, M. (2016). La educación a distancia virtual: Desarrollo y características en cursos de matemáticas. Revista Virtual Universidad Católica del Norte, 48, 33-55 http://revistavirtual.ucn.edu.co/index. $\mathrm{php} /$ RevistaUCN/article/view/760/1286. 
Terreni, L., Vilanova, G., Varas, J. (2019). Desarrollo de competencias digitales en propuestas pedagógicas en ambientes mediados: Un caso en educación superior bajo modelo de aula extendida. Informes Cientificos Técnicos - UNPA, 11(3), 61-87. https://doi.org/10.22305/ict-unpa.v11. n3.797.

Torres García, A. J., Ochoa Adame, G. Li. (2018). Desigualdad salarial asociada al uso de las TIC en México: Un análisis por ocupaciones. Cuadernos de Economía, 37 (74), 353-389. https://doi.org/10.15446/ cuad.econ.v37n74.56549.

Varela, R., Castillo, R. A., y Osegueda, J. M. (2013). El empleo formal e informal en México: Un análisis discriminante. Papeles de población, 19(78),111-140.http://www.scielo.org.mx/scielo. php?script=sci_arttext\&pid=S140574252013000400006\&lng=es\&tlng=es. 